\title{
A Rare Case of Peritoneal Sarcoidosis in a 36-Year-Old Construction Worker
}

\author{
Saman Chubineh Kyle Katona \\ North Shore University Hospital, Manhasset, N.Y., USA
}

\section{Key Words}

Peritoneal sarcoidosis - Peritoneal carcinomatosis · CA-125 level · Asbestos exposure

\begin{abstract}
A 36-year-old man was admitted for persistent abdominal pain and weight loss. He had had significant exposure to asbestos, as he had been active in cleanup after the World Trade Center attack. A CT scan of his abdomen was initially read as peritoneal carcinomatosis without evidence of ascites. Infectious etiology was subsequently ruled out and diagnostic laparoscopy was performed for tissue diagnosis revealing noncaseating granulomas consistent with peritoneal sarcoidosis. Of note, he had an elevated CA-125 level on admission, which is unique in males with peritoneal sarcoidosis.
\end{abstract}

\section{Introduction}

Sarcoidosis is a chronic, multisystem disorder of unknown etiology, characterized by an accumulation of noncaseating granulomas and involvement of any organ [1]. Serosal sarcoidosis is rarely reported, especially when it is the unique manifestation of the disease [2]. We present a rare case of peritoneal sarcoidosis in a man who presented with abdominal pain and a diagnosis of peritoneal carcinomatosis on CT scan.

\section{Case Report}

A 36-year-old man with no significant past medical history presented to our emergency department with a chief complaint of abdominal pain for one month. The abdominal pain had initially localized to the left lower quadrant, but had recently progressed to involve the entire abdomen. Associated symptoms included an unexplained 20-pound weight loss over the previous two months, fever/night sweats, loss of appetite, and nausea without vomiting. Physical exam revealed a thin man in no acute distress with a soft but diffusely tender abdomen. Recent workup included endoscopy revealing gastritis and colonoscopy showing internal hemorrhoids and a $5 \mathrm{~mm}$ polyp in the descending colon. A CT scan of the abdomen showed extensive peritoneal carcinomatosis without evidence of ascites (ig. 1 ). 
Of note, this patient had an eight pack year smoking history from the age of 17 to 21 years, when he smoked two packs per day. He had also experienced extensive dust/asbestos exposure as he had been involved in construction and had worked for an asbestos removal agency for five years, ten years prior to his presentation. In addition, he had been active in the cleanup after the World Trade Center attack, spending hours daily at Ground Zero. He had no respiratory complaints at any time.

CT scan of the chest was within normal limits, without evidence of hilar adenopathy. Laboratory values were significant for anemia with a CBC revealing a WBC of $6.4 \times 10^{3} / \mu \mathrm{l}$, hemoglobin of $9.9 \mathrm{~g} / \mathrm{dl}$ and hematocrit of $30 \%$ (MCV of 85 ), and platelets of $654 / \mu$. The differential on the WBC included $85 \%$ neutrophils and $9 \%$ lymphocytes. Of note, the patient also had elevated liver enzymes with AST of $89 \mathrm{IU} / \mathrm{l}$, ALT of $112 \mathrm{IU} / \mathrm{l}$, and alkaline phosphatase of $190 \mathrm{IU} / \mathrm{l}$. Tuberculosis workup was negative and tumor markers were significant for an elevated CA-125 of $126 \mathrm{U} / \mathrm{ml}$ (four times the upper limit of normal). Surgery was consulted and a diagnostic laparoscopy was performed for tissue diagnosis. Omental adhesions were visualized and biopsies were obtained, which were sent for frozen section and read as diffuse inflammatory changes. Due to lack of diagnosis, the procedure was converted to an open procedure. No pathology was noted on the peritoneum; however omental caking was isolated and sent for frozen section that also revealed inflammatory changes. Acid-fast stain for mycobacterium and all other cultures was negative. No malignant cells were noted.

The patient was discharged after an uneventful postoperative course. Final histology revealed diffuse inflammatory changes with noncaseating granulomatous disease consistent with peritoneal sarcoidosis. The patient was discharged without steroids and his abdominal pain eventually resolved. He also regained approximately ten pounds over the next four weeks.

\section{Discussion}

On presentation, our differential diagnosis was peritoneal carcinomatosis, infectious etiology, namely fungal and tuberculous, and peritoneal mesothelioma based on the patient's extensive asbestos exposure (although unlikely given his exposure of only ten years). While sarcoidosis can affect any organ system within the body, serosal involvement has rarely been reported [2]. A literature search from January 1966 to February 2008 using the MEDLINE database revealed nineteen cases reported. This makes our new case the 20th.

Elevated CA-125 levels have been noted in reported cases of peritoneal sarcoidosis, and a link to symptoms mimicking ovarian carcinoma has been described [3]. Five cases have been reported to have an elevated CA-125 level, all female [3]. To our knowledge, this is the first reported case of a man presenting with peritoneal sarcoidosis with an elevated CA-125 level. It is unknown whether the other reported cases had CA-125 testing performed. CA-125 has been reported to be elevated in certain granulomatous conditions [3]. Thus, a possible correlation exists between peritoneal sarcoidosis and the glycoprotein CA-125, although the role of gender in the etiopathogenesis remains unclear.

Therefore, we suggest that situations in which causes of peritoneal nodules are excluded, including infectious and malignant, peritoneal sarcoidosis should be considered. In addition, while workup can include serum angiotensin converting enzyme levels, calcium, and tuberculin skin tests, CA-125 level should also be considered. Whether exposure to asbestos was related to the development of sarcoidosis in this patient is uncertain and no such correlation could be found in reviewing the literature. 

\begin{tabular}{l|l} 
Case Reports $h$ & $\begin{array}{l}\text { Case Rep Gastroenterol 2008;2:369-372 } \\
\text { D0l: 10.1159/000158544 }\end{array}$
\end{tabular}

Fig. 1. CT scan of the abdomen showing extensive peritoneal seeding.

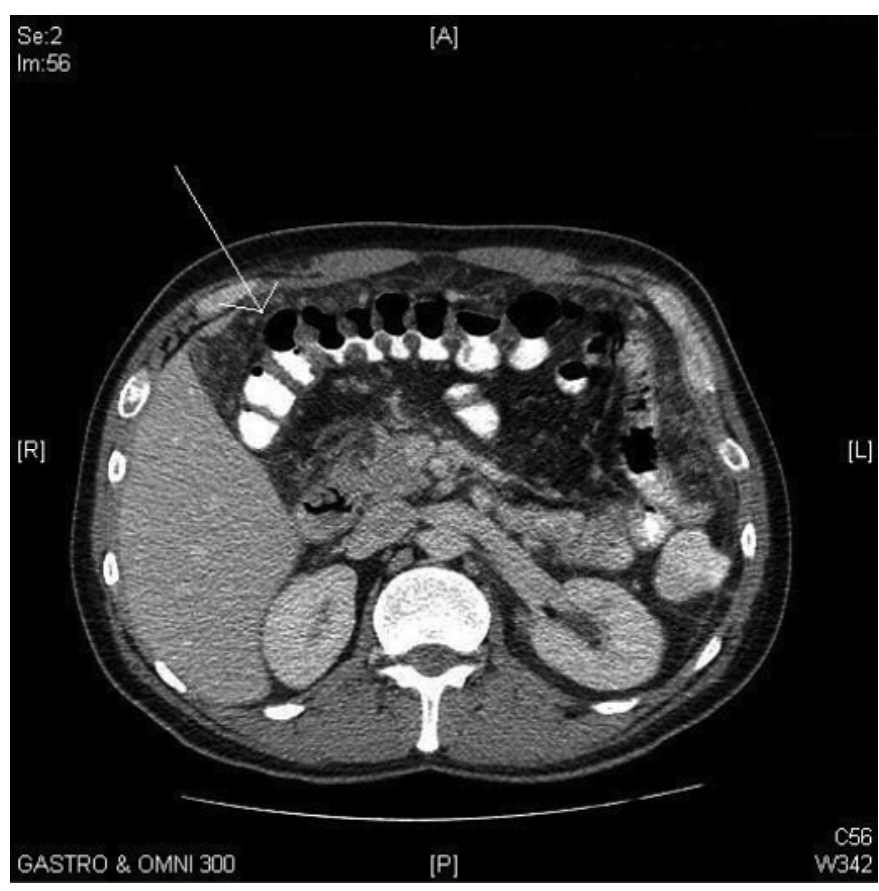




\section{References}

1 Baughman RP, Lower EE, du Bois RM: Sarcoidosis. Lancet 2003;361:1111-1118.

2 Uthman IW, Bizri AR, Khalifeh MJ: Peritoneal sarcoidosis. Semin Arthritis Rheum 2002;31:353.

-3 Kalluri M, Judson MA: Sarcoidosis associated with an elevated serum CA 125 level: description of a case and a review of the literature. Am J Med Sci 2007;334:441-443. 